\title{
Research on Robot Motion Control based on Artificial Potential Method
}

\author{
GuangBing Zhou ${ }^{1,}$, Huan Lei ${ }^{1, b}$, LiangSheng Wu ${ }^{1, c}$ \\ ${ }^{1}$ Guangdong key Laboratory of modern control technology of Guangdong Institute of Intelligent \\ Manufacturing, Guangzhou, China
}

Keywords: Artificial Potential Field; Robot; Angular Deflection Method; Simulation Control

\begin{abstract}
Humanoid robot is a kind of rare multiple-degree-of-freedom system with high order, non-linearity and nonholonomic constraint in project and it has powerful kinematic dexterity, which therefore has provided a pretty ideal experiment platform for the kinematics, dynamics, simulation technique, multi-sensor fusion and control theory and other researches of robot. In this paper, a new kind of angular deflection method is provided to make robot quickly escape from the local minimum point, which has planned a smooth collision-free path successfully. And simulation experiment is used to prove the effectiveness of the method planning.
\end{abstract}

\section{Introduction}

Aiming at the characteristic that humanoid robot has numerous driving mechanisms and sensing devices, this Paper synthesizes the advantages of centralized distributed systems and proposes a kind of improved motion control system structure of humanoid robot, which is composed of synergy movement treatment module, data communication module and quadruped locomotion control module. And inside of each basis motion control module includes joint controller, joint driver and several sensors. The structure has fully taken into account the situation that joints in close position and similar functions of humanoid robot may be tightly coupled and has carried out appropriate concentration of functions, which not only retains the intelligent and flexible advantage of distributed structure but also reduces the burden of data communication so as to adapt to the control and sensing requirements of humanoid robot in a better manner [1-5].

\section{Model of artificial potential field algorithm}

The gradient potential field method is commonly used as a potential field method. The robot has a certain abstract potential energy in the potential field and its negative gradient direction indicates the direction of the abstract force that the robot system suffers from, which is the very force that causes the robot to bypass the obstacle and advance towards the target.

Assuming that the two-dimensional working space of the robot is $\mathrm{W}=[x, y]^{T}$, and the construction of potential field is the application of gravity and repulsion to the robot (in the potential field construction, the subscript o denotes the obstacle and the subscript a denotes the target point):

$$
U(W)=U_{a}(W)+U_{o}(W)
$$

Of which, $U_{a}(W)$ is the gravitational field and $U_{o}(W)$ repulsion field. Therefore, the resultant force of robots in the potential field can be expressed as:

$$
\begin{aligned}
& F=F_{a}+F_{o} \\
& F_{a}=-\operatorname{grad}\left[U_{a}(W)\right], F_{o}=-\operatorname{grad}\left[U_{o}(W)\right] 。
\end{aligned}
$$

Of which, $F_{a}=-\operatorname{grad}\left[U_{a}(W)\right]$ is for gravity and $F_{o}=-\operatorname{grad}\left[U_{o}(W)\right]$ is for repulsion.

The resultant force that robot suffers from in the two-dimensional space is: 


$$
\nabla U(W)=\left[\begin{array}{l}
\frac{\partial U}{\partial x} \\
\frac{\partial U}{\partial y}
\end{array}\right]
$$

The gravitational potential function for target to robot is:

$$
U_{a}(W)=\frac{1}{2} \alpha\left(W-W_{a}\right)^{2}
$$

Of which, $\alpha$ is gravitational gain coefficient, $W$ is the current coordinate of the robot in the two-dimensional space, $W_{a}$ is the position of target point, and $\left(W-W_{a}\right)$ is the relative distance from the robot to the target point, and corresponding gravity can be translated into $F_{a}=-k\left(W-W_{a}\right)$.

Repulsion potential function of obstacles is defined as:

$$
U_{o}(W)= \begin{cases}\frac{1}{2} \beta\left(\frac{1}{\rho}-\frac{1}{\rho_{0}}\right)^{2} & \text { if } \rho \leq \rho_{0} \\ 0 & \text { if } \rho>\rho_{0}\end{cases}
$$

Of which, $\beta$ is repulsion gain coefficient, $\rho_{0}$ is a constant and denotes the influential distance of obstacle, $\rho$ is the shortest distance from the robot to the obstacles. And corresponding repulsion is:

$$
F_{o}=-\operatorname{grad}\left[U_{o}(W)\right]= \begin{cases}\beta\left(\frac{1}{\rho}-\frac{1}{\rho_{0}}\right) \frac{1}{\rho^{2}} \frac{\partial \rho}{\partial W} & \text { if } \rho \leq \rho_{0} \\ 0 & \text { if } \rho>\rho_{0}\end{cases}
$$

Of which, $\frac{\partial \rho}{\partial W}=\left[\frac{\partial \rho}{\partial x}, \frac{\partial \rho}{\partial y}\right]^{T}$.

When the robot encounters several obstacles, superposition of potential fields is used to obtain the repulsion potential field of several obstacles to the robot.

\section{Robot Motion Control of Improved Artificial Potential Field Algorithm}

\section{Solving target unreachable}

The main reason for the existence of the target unreachable problem is that when the target is within the influence scope of the obstacle, the global minimum point of the whole potential field is not the target point. Because when the robot approaches the target, the potential field of the obstacle increases rapidly, causing the robot to move away from the target point. As shown in Fig. 1 (a) (the blue circle in the figure is the obstacle in the set environment; the red dot is the path point that the robot has walked through; and the small triangle is the target point), if the repulsion field tends to zero when the robot approaches the target, then the target point will be the global minimum point of the whole potential field. Therefore, the relative distance between the robot and the target point shall be also considered in defining the function of repulsion potential field so as to establish a new function of repulsion potential field.

The improved repulsion potential field function is:

$$
U_{o}(W)= \begin{cases}\frac{1}{2} \beta\left(\frac{1}{\rho}-\frac{1}{\rho_{0}}\right)^{2} \rho_{a}{ }^{n} & \text { if } \rho \leq \rho_{0} \\ 0 & \text { if } \rho>\rho_{0}\end{cases}
$$

Of which, $\rho_{a}=\left\|W-W_{a}\right\|$, denoting the relative distance from the target object to the robot. $n$ is an arbitrary real number more than zero.

The corresponding repulsion of the robot in the potential field is: 


$$
F_{o}=-\operatorname{grad}\left[U_{o}(W)\right]= \begin{cases}F_{o 1} N_{1}+F_{o 2} N_{2} & \text { if } \rho \leq \rho_{0} \\ 0 & \text { if } \rho>\rho_{0}\end{cases}
$$

Of which:

$$
\left\{\begin{array}{l}
F_{o 1}=\beta\left(\frac{1}{\rho}-\frac{1}{\rho_{0}}\right) \frac{\rho_{a}^{n}}{\rho^{2}} \\
F_{o 2}=\frac{n}{2} \beta\left(\frac{1}{\rho}-\frac{1}{\rho_{0}}\right)^{2} \rho_{a}{ }^{n-1}
\end{array}\right.
$$

In the improved repulsion potential field function, a regulatory factor $\rho_{a}{ }^{n}$ is added; when the gravitational potential field increases, the repulsion potential field $U_{o}(W)$ decreases, causing the overall potential field become minimum only at the target point, as shown in the Fig. 1(b):

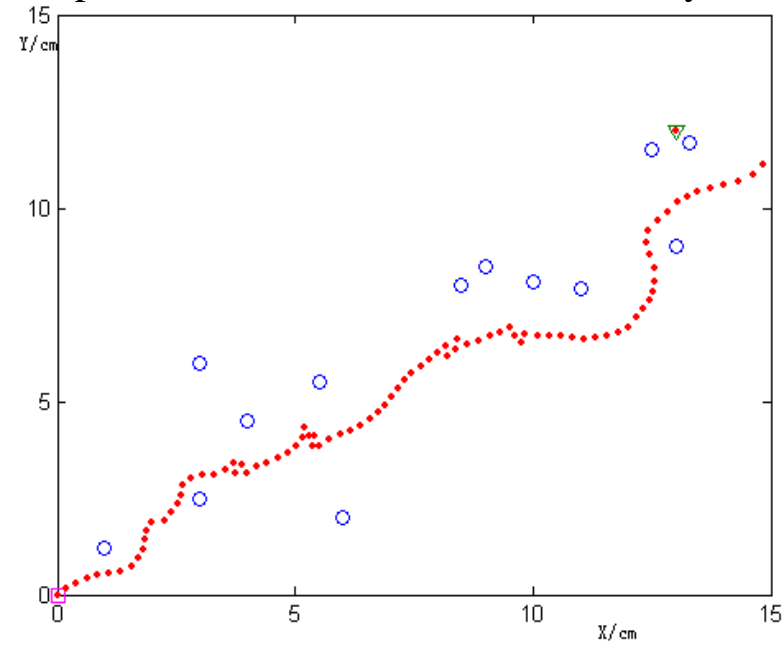

(a) Target unreachable path

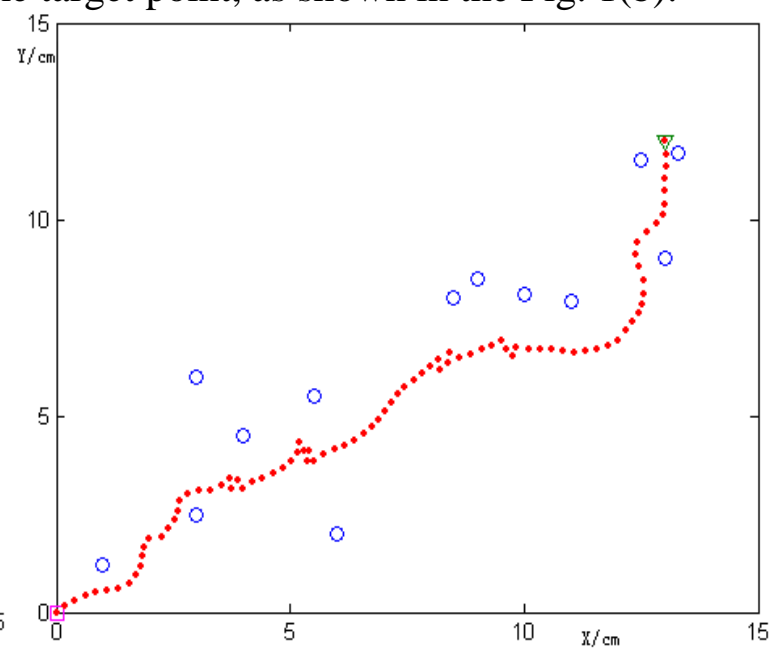

(b) Target reachable path

\section{Solving local minimum}

Fig.1. Artificial potential field method used to realize target reachable

During the process that a mobile robot looks for a path, there may be a special case - falling into a local minimum point. When a robot performs a path search, if one or more obstacles occur between the robot and the target, and the repulsion of obstacles on the robot is equal to the gravity that the robot suffers from and both of them are at the same straight line, then the resultant potential field force that the robot suffers from will be $F=F_{a}+F_{o}=0$, at the moment of which, the robot will stop advancing and fall into a local minimum point, resulting in the path planning failure.

In this Paper, an angular deflection method is used to solve the problem that the robot falls into the local minimum point. When the robot falls into the local minimum point, an angular deflection is given to the robot to change the motion direction the robot: along the direction of connecting the current position of the robot and the target point, deflects clockwise an angle of $\left(0 \sim \frac{\pi}{2}\right)$ and regard the direction after an angle of $\frac{k \pi}{2}$ as the direction of next motion of the robot, of which the $\mathrm{k}$ value is taken as the real number gradually increased within $(0 \sim 1)$. If the robot can walk away from the local minimum point after a turn of an angle, then the robot will continue to look for the path to the target under the action of the potential field force; if the robot fails to walk away from the local minimum point, then a continuously increased deflection angel is given to the robot until it escapes from the local minimum point. During the advancing process of the robot, step $l$ is still the one in initialization. The planning process chart of above algorithm path is shown in Fig. 2.

In the simulation experiment, the Paper sets the working environment as a two-dimensional space of $15 \times 15$, of which, the origin $[0,0]$ is set as the initial position of the robot, the point $[13,12]$ is set as the target point, and the number of obstacles $\mathrm{N}$ is set as 17 , the gain coefficient $\alpha$ of 
gravitational potential field is set as 2 , the gain coefficient $\beta$ of repulsion potential field is set as 5 , and the robot step is $l=0.25$. When the robot falls into a local minimum point, the resultant force of repulsion force and gravity is zero, at the moment of which, a deflection angle of $\frac{k \pi}{2}$ is given to the robot to change the motion direction of it to find the next position and determine whether it escapes from the local minimum point at the moment. If it still fails to escape, continue to change the $\mathrm{k}$ value until it successfully escapes from the local minimum point.

Fig. 3 (a) shows the traditional path planning in the literature before the improved algorithm, where the robot falls into the local minimum point and cannot plan out a path reaching the target point. Fig. 3 (b) is a path diagram of the robot to escape the local minimum point after the improved algorithm. And the experiment shows that the robot is able to effectively escape from the local minimum point and plans out a path reaching the target point by using the new algorithm provided in the Paper.

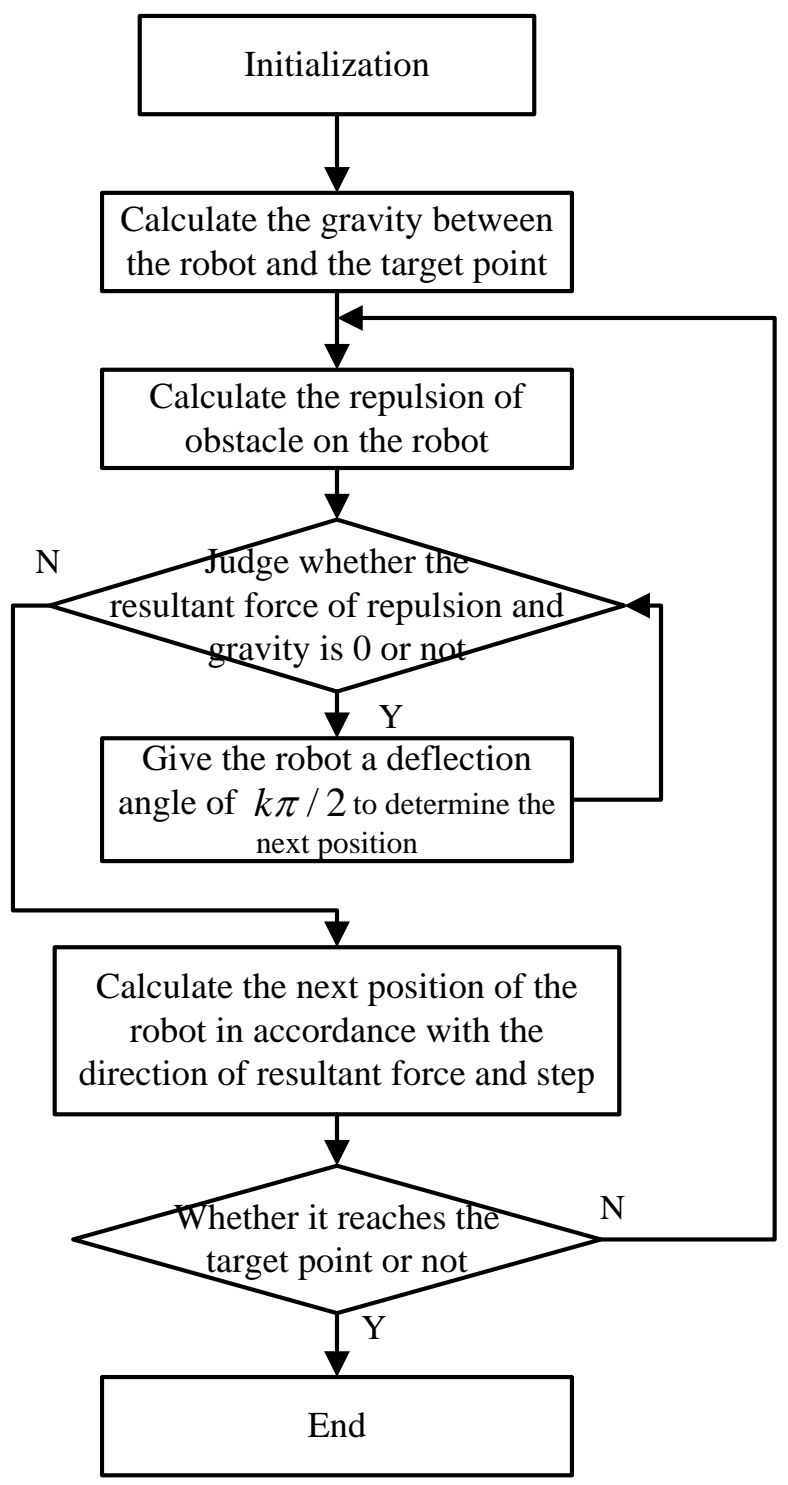

Fig.2. Path planning flow chart of improved algorithm 

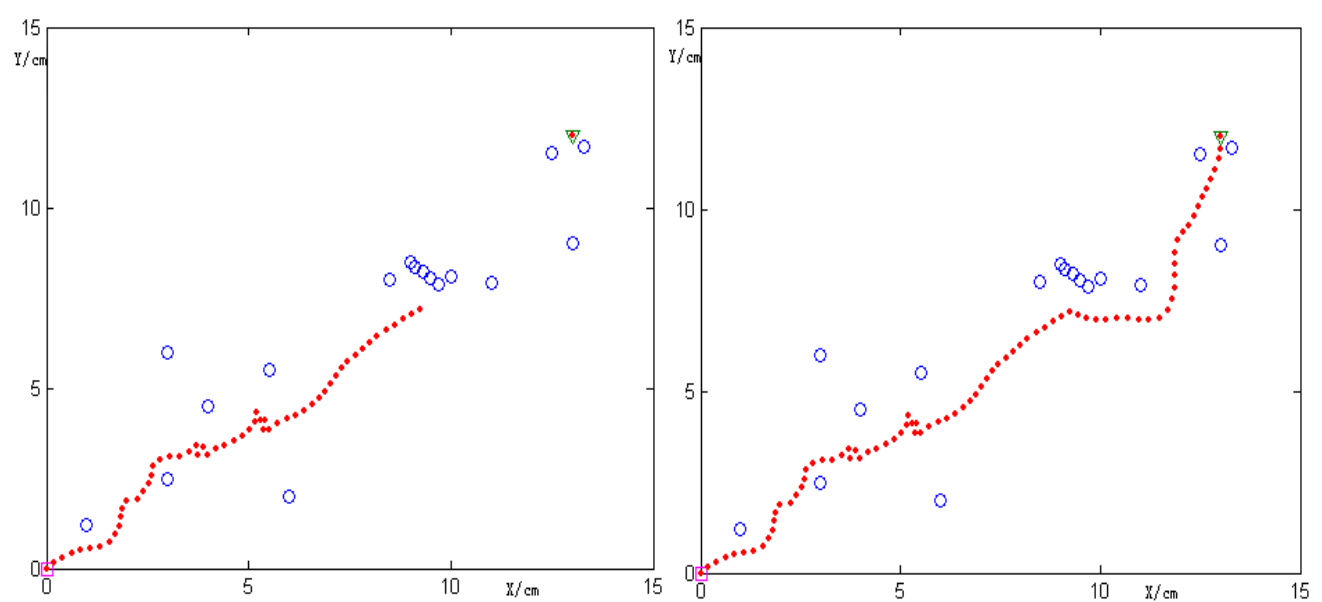

(a) Path of robot to fall into local minimum point (b) Path of robot to escape from minimum point after improved algorithm

Fig.3. Path of robot to fall into local minimum point and escape from it

\section{Conclusion}

Humanoid robot experiment platform has been tested in multi-joint cooperative control and the experiment result shows that hardware and software design of the system can meet the basic requirements of a robot's motion behavior and can realize a considerable control precision. This Paper is based on the traditional artificial potential field method with local minimum point problem which leads to path planning. When a number of small obstacles gather between (or large obstacle block between) the robot and the target object, the robot is easily caught in the local minimum point; through the use of the method of increasing the angular deflection, the robot is able to escape from the minimum point and moves towards the target point rapidly by bypassing the obstacle, thus solving the problem that the robot cannot find the path and saving the planning time. And the simulation result has also proved the effectiveness of above method.

\section{Acknowledgement}

Supported by Guangdong Province Science and Technology Project(2016B090926005; 2016B090926002)

\section{References}

[1] Peters E E (2015) Fractal market analysis: applying chaos theory to investment and economics [J]. Chaos Theory, 34(2):343-345.

[2] Tripodi A; Primignani M; Chantarangkul V; Dell'Era A; Clerici M; de Franchis R; Colombo M; Mannucci PM (2013) Performance Evaluation of Spreading Codes with Negative Auto-Correlation Based on M-sequences and Chaos Theory[J]. Ieice Technical Report Circuits \& Systems, 112(6):5-10.

[3] Wang J, Shi Q (2013) Short-term traffic speed forecasting hybrid model based on Chaos-Wavelet Analysis-Support Vector Machine theory[J]. Transportation Research Part C Emerging Technologies, 27(2):219-232.

[4] Peng J, Hampton J, Doostan A (2013) A weighted L1-minimization approach for sparse polynomial chaos expansions[J]. Journal of Computational Physics, 267(5):92-111.

[5] Migliorati G, Nobile F, Schwerin E V, et al. (2014) Analysis of Discrete $\backslash\left(L^{\wedge} 2 \backslash\right)$, Projection on Polynomial Spaces with Random Evaluations[J]. Foundations of Computational Mathematics, 14(3):419-456. 